\title{
Voice Based Email with Security for Visually Challenged
}

\author{
Latha L, Babu B, Sowndharya S
}

\begin{abstract}
Communication is an important aspect of connectivity among the people of different countries. Various communication technologies include telephone, smart phone, internet applications such as email, what's app, sms, etc. These technologies are integrated with the internet. Letters were a form of communication in olden days. They were replaced by Email (also known as electronic mail) which is a form of dual way communication. In email, either two persons can communicate privately or group mails can also be forwarded. But there is a problem that these internet integrated technologies are workable only with visual perception. There is an estimation that there are about 20 million around the world who are visually challenged. Technology development is not only meant for normal people. The main aim of this project is to provide an android application specially developed for the visually challenged people to send and receive mails. To ensure privacy on sending the message, a hardware device with human detection sensor is arranged that can be embedded into the phone using other technology such as nanotechnology.
\end{abstract}

Keywords- Android, Recognizer intent, TTS, Gmail and Google map, PIR sensor, human presence detection.

\section{INTRODUCTION}

In early days visually challenged people were not able to use the computer due to the reasons that they face due to visual perception. They could not see the visual perspective of the input or output from the computer. Traditional forms of accessing internet are more difficult for visually challenged than the normal people. But nowadays many researches are undergoing to develop a fine technology that enhance the skills of the visually challenged persons to use the recent technologies in internet apart from the basic knowledge about the computers. According to WHO, it is estimated that around 180 million people are visually disabled out of which 40 to 45 million people are blind. Hence an initiative to provide them an access to the internet were introduced. In this project, an interactive UI is made to access their mail easily.

\section{LITERATURE SURVEY}

On analyzing similar proposals and research papers regarding the voice based email, the technologies commonly used in those systems are:

Screen Readers [8]: Screen readers are software programs that allows the users to read the contents on the screen using speech synthesizer. It is an interface between computer's

Revised Manuscript Received on 14 August, 2019.

Latha L, Professor, Kumaraguru College of Technology, Coimbatore, Tamil Nadu, India.(Email: latha.1.cse@kct.ac.in)

Babu B, UG students, Kumaraguru College of Technology, Coimbatore, Tamil Nadu, India, (Email: babu.15cs@kct.ac.in)

Sowndharya S, UG students, Kumaraguru College of Technology, Coimbatore, Tamil Nadu, India.(Email: sowndharya.15cs@kct.ac.in) operating system and its application and the user. It makes the user to enter the commands that should be said by the speech synthesizer. The user can locate the cursor, focus on the text, read a location in the map and many more activities can be performed.

Braille Keyboards [7]: Braille computer keyboards are very rare. They are connected to the computer similar to a standard computer keyboard. It accepts the input from it. They are also used in Braille Typewriters.

IVR [3]: It is an Interactive Voice Response system. It allows the user to interact with the system through voice. It analyses and synthesizes the user's voice and reply back to them in the form of voice, text, email, etc.

Speech to text conversion: The given voice input is taken to the server where it get converted as text output.

Text to speech conversion: The working is similar to the previous one but the output response will be in the form of voice.

The disadvantages of the existing system are as follows:

1. Screen readers cannot spell technical and biological terms.

2. Braille language must be known and when using keyboards shortcuts needs to be notified.

3. Fingerprint authentication can be easily acquired through any malpractices.

4. Since the systems are web and desktop applications they cannot be used by visually challenged without some help.

These disadvantages are tried to be eliminated in the proposed system.

\section{III.PROPOSED SYSTEM}

The proposed system focuses on providing the basic functionalities like composing, reading, sending and receiving emails along with voice based interaction. This facilitates working with each of the above features as well as the provision for sending text as well as voice based email.

The proposed system allows blind people to use Email system easily. As the input to the system does not use keyboard or mouse, users can easily give input by speaking the message.

The proposed system is an android application which enables the blind users to access their mail without the help of third persons. The initial steps in which they require the help of others are in the installation of the app and the registration of the user. The registration is mandatory for all the users, so that the user will be always connected further for future mail communications. The techniques used to implement the voice based email are: 


\section{VOICE BASED EMAIL WITH SECURITY FOR VISUALLY CHALLENGED}

\section{Speech to text conversion in android:}

Android provides options to the interfaces the for recognition listener that is used for receiving the speech from the user. First, recognizer intent is created. It takes the user's speech input and returns it to the same activity. In case of language, the android speech recognizer provides options for the user to say the input in 10 different languages. Then the speech response is to be caught on some activity or same activity. The code converts the response to text and displays and or again send as an input to the text to speech converter.

\section{Text to speech conversion in android:}

It is an integral part of the application. It reads the text and converts it into the audio and delivered to the user via microphone of the user. Android introduced and supports this TTS feature especially for visually challenged and visually impaired people. An object is created in a class to get the text. Android TTs engine supports many languages. The text is passed as a parameter to the speech conversion function. In the listener function, the converted text is sent as an inbuilt voice to the user.

API's and keys:

The system were implemented in android. The android provides packages such as recognizer intent and TTS to convert these speech to text and text to speech. Additionally api key to access Gmail and Google map has to be obtained. They provide web services to access mails and obtain current location of the user.

\section{Human presence detection system [9]:}

The components are connected in such a way that whenever there is a motion identified by the PIR sensor in its surrounding for 6 meters then both LED and buzzer gets turned on. As a result LED glows and buzzer starts ringing. The prototype cannot be replaced in mobile phone. Since the size of sensor is pretty big. Many Micro Electro Mechanical Elements are being embedded in smart phones nowadays. So if there is a possibility to reduce this sensor or developing an application that gets the result of sensor as a digital information and starts alarming in the phone. The person presence identification will be very useful for visually challenged people in many aspects. The prototype developed is to demonstrate the working of the motion capture sensor. But the static person or object cannot be identified using the PIR sensor. So this sensor has to be replaced by any other sensor in order to receive accurate result.

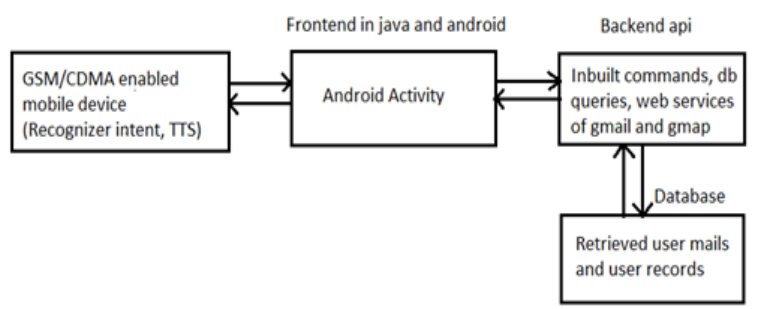

Fig.1. Dataflow diagram

\section{IV.RESULTS AND DISCUSSION}

If the user is using this app for the first time they have to register so that the google automatically retrieves mail from the user account. The registration page is as follows. The registration is mandatory for all the users to ensure that they have an email account so that the mail communication will be possible. After registration, the page directs to the main user interface where the user can provide their own option like send mail, setting alarm, location finding, etc.

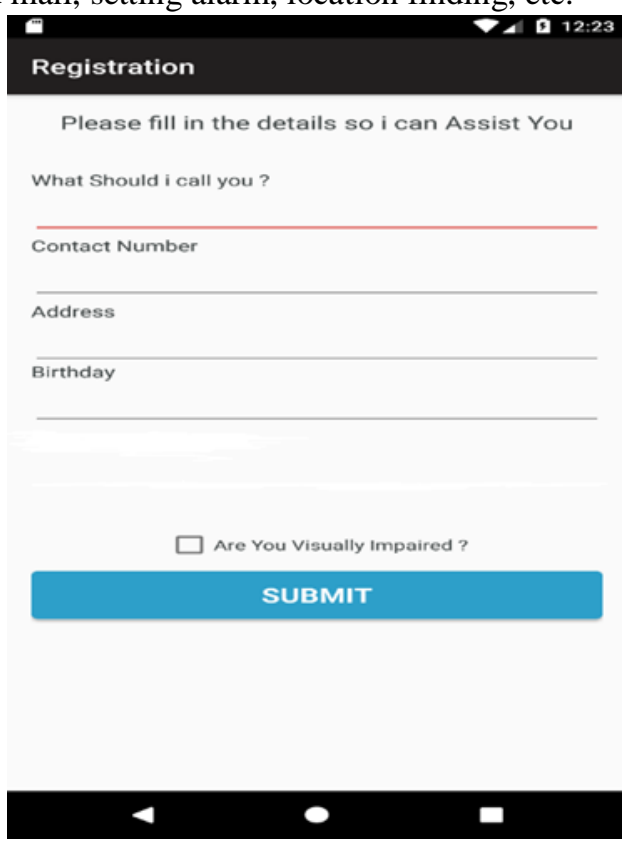

Fig.2. Registration page

On saying email, the mail module gets opened. Tap once to say help and know the options available with the mail module. Options such as writing mail, getting mail, and search for mail are available with this module.

The following images shows that on user saying email to the application the mail module starts executing. To proceed further again help should be said to the app and it displays the specific help module for the mail. It shows options such as

1. Compose mail: To write mail which prompts for the receiver's mail id and content or message from the sender.

2. Search mail: Leads to search for a particular mail using any keyword or name of the sender.

3. Get mails: Retrieves first three mails from the inbox and starts speaking them. 


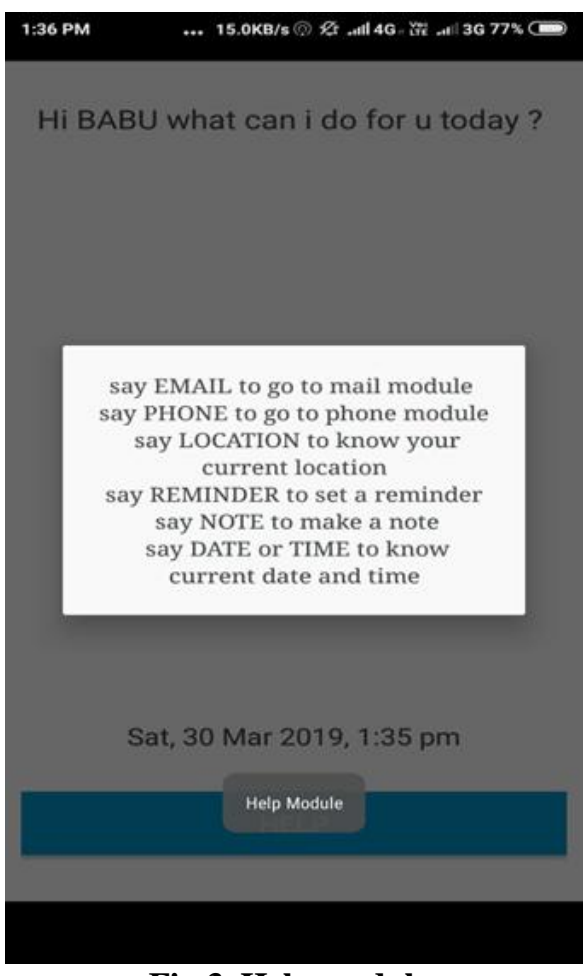

Fig.3. Help module

On saying phone, the phone module will be executed and the user can mention the contact name of the person whom they want to call. The api connects with the call and they can communicate with them.

The app interconnects with the telecom subsystem of the user's device. The framework manages the audio and video routing. The service that provides permission to access that subsystem is executed and the service has been declared. The service has been binded with the subsystem. As a result, on hearing the command call the service gets executed. The app also searches for the contact name in the user's phone and makes call. The framework establishes the connection between the sender and receiver.

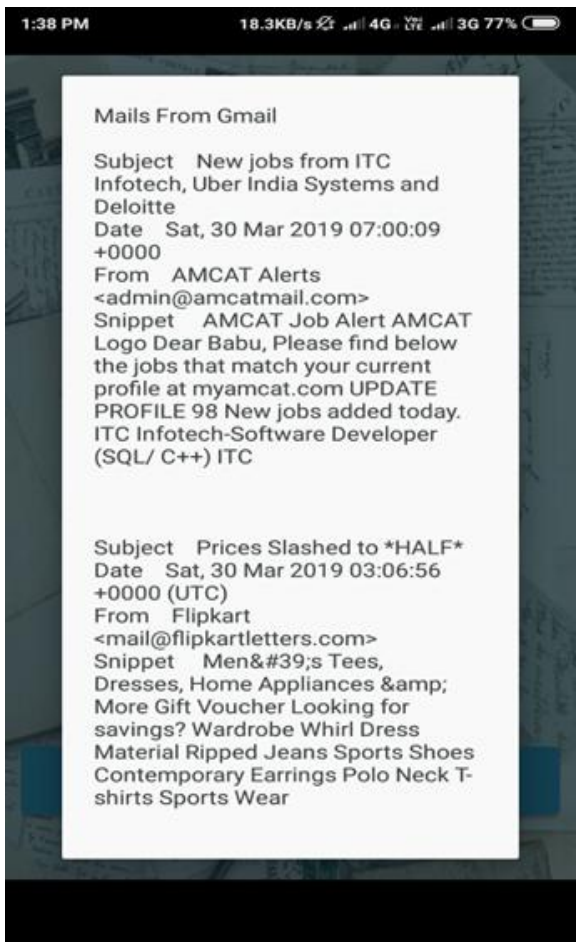

Fig.4. Getting mail through api

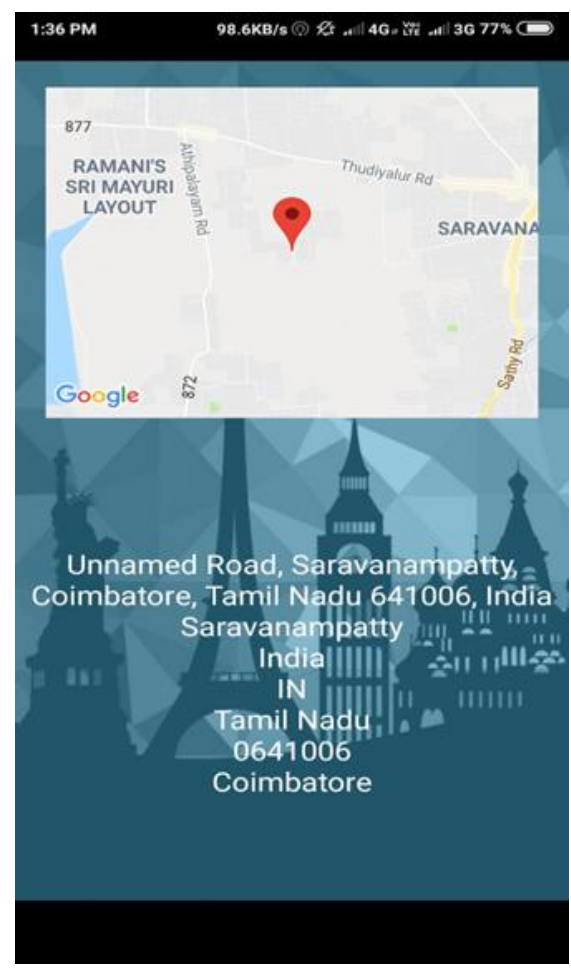

Fig.5. location module

While using this application in order to ensure that there are no intruders nearby, this hardware set up has been arranged.

1. PIR sensor: To identify the presence of human.

2. Buzzer and LED: To alert the user.

3. Arduino: To load the output program and organize the working

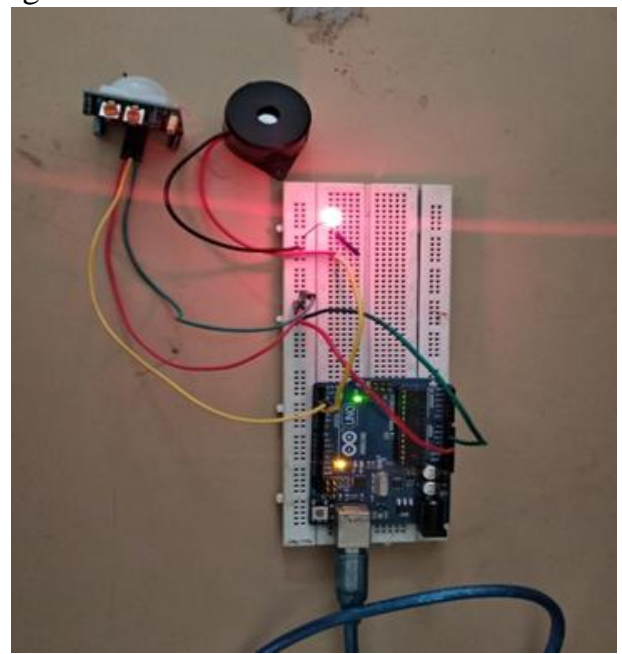

Fig.6. Circuit for human presence detection with PIR sensor

\section{V.CONCLUSION}

The application was developed with the aim that it can be used by both visually challenged and normal sighted people. Screen readers, Braille keyboards, etc., were useful for their studies. But when it comes to searching or using an application, it is bit difficult. Hence this application can be used to send and receive mail from the sender and receiver.

Published By

Blue Eyes Intelligence Engineering \& Sciences Publication

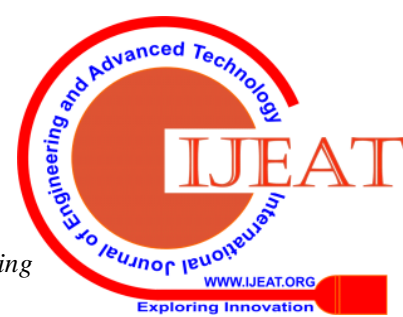


To ensure privacy of sending message, a hardware to identify the presence of human has been arranged which can be made using Micro Electro Mechanical Elements. This will be very useful in comparison with the other applications of visually challenged.

\section{FUTURE SCOPE}

Many researches are undergoing regarding the usage of internet by the visually challenged. In the total population of the world there are about $60 \%$ of visually challenged in India. The literacy rate of visually challenged is also getting high. Hence it is necessary to make developments in the visual perception of internet to make them use the internet as like normal person. Currently available systems such as Screen readers, Interactive voice Response, etc. also requires some visual perception by disabled to create account and to use them. An interactive voice based email system can help visually challenged to communicate easily in this modern world. To ensure privacy there are systems to identify human presence. Many sensors such as accelerometer, gyrometer are getting embedded in smart phones as Micro Electro Mechanical Elements hence they are embedded. If person identification sensor is also able to embed in smart phone specially designed for visually challenged, then it will be very useful for them to use email and other applications.

\section{REFERENCES}

1. Dhanashree. D. Zope, Pooja. B. Nevewani, Pooja. G. Teje, Nusrat Parveen (2017), "Voice Based E-Mail System for Blind People", in International Journal of Scientific Research in Computer Science and Engineering, Vol.5, Issue.4, pp. 73-75.

2. Carmel Mary Belinda M.J, Rupavathy.N, Mahalakshmi N R (2018), "A Voice Based text mail system for visually impaired", in International Journal of Engineering and Technology, 7 (1.7) pp. 132-136.

3. K. Jayachandran, P. Anbumani (2017), "Voice Based Email for Blind People", in International Journal of Advanced Research, Ideas and Innovations In Technology, Vol.3, Issue.3, pp. 1065-1071.

4. Tharani K K, Shalini R, Jeyanthi I, Dr.Deepalakshmi R (2017), "Voice Based Mail Attachment For Visually Challenged People", in International Journal of Scientific and Engineering Research, Vol.8, Issue.5, pp. 126-130.

5. Saurabh Sawant, Amankumar Wani, Sangharsh Sagar, Rucha Vanjari, M R Dhage (2018), "Speech Based Email System for Blind and Illiterate People", in International Research Journal of Engineering and Technology, Vol.5, Issue.4, pp. 2398-2400.

6. G. Shoba, G. Anusha, V. Jeevitha, R. Shanmathi (2014), "An Interactive Email for Visually Impaired", in International Journal of Advanced Research in Computer and Communication Engineering, Vol.3, Issue.1, pp. 5089-5092.

7. Pradeep Manohar, Aparajit Parthasarathy, "An Innovative Braille System Keyboard for the Visually Impaired".

8. G. Tejaswani, Afroz.B, Prof. Sunitha S (2016), "A Text Recognizing Device For Visually Impaired", in International Journal of Engineering and Computer Science, Vol.7, Issue.3, pp. 23697-23700.

9. B. Ivanov, H. Ruser, M. Kellner, "Presence detection and person identification in Smart Homes". 